\title{
Toeplitz algebras and spectral results for the one-dimensional Heisenberg model
}

\author{
Mondher Damak ${ }^{1}$, Marius Măntoiu ${ }^{2}$ \\ and Rafael Tiedra de Aldecoa ${ }^{3}$ \\ 1 Département de mathématiques, Université de Sfax, B.P. 802- \\ 3018, Sfax, Tunisia \\ 2 Institute of Mathematics "Simion Stoilow" of the Romanian Academy, \\ P. O. Box 1-764, Bucharest, RO-014700 \\ 3 Département de mathématiques, Université de Paris XI, 91405 \\ Orsay Cedex France \\ E-mails: Mondher.Damak@fss.rnu.tn, Marius.Mantoiu@imar.ro, \\ rafael.tiedra@math.u-psud.fr
}

\begin{abstract}
We determine the structure of the spectrum and obtain non-propagation estimates for a class of Toeplitz operators acting on a subset of the lattice $\mathbb{Z}^{N}$. This class contains the Hamiltonian of the one-dimensional Heisenberg model.
\end{abstract}

\section{Introduction}

Spectral theory of Toeplitz operators and its connection with $C^{*}$-algebras is a vast topic. We only indicate Dou72] as a textbook systematization of part of the early theory. Since the appearence of Douglas' book, the theory has evolved by extension and abstraction in many directions. The works closest to the present paper are the ones relating Toeplitz operators and Toeplitz algebras to ordered groups Mur87, Mur91, Xu99, XC99.

Our interest in the topic has been aroused by the remark Str67, Str74, RS78, that the one-dimensional Heisenberg Hamiltonian $H$ of ferromagnetism can be written as a direct sum $H=\oplus_{N \in \mathbb{N}} H_{N}$, where $H_{N}$ can be interpreted as the Laplace operator on the subgraph

$$
\mathbb{Z}_{<}^{N}:=\left\{\left(x_{1}, \ldots, x_{N}\right) \in \mathbb{Z}^{N}: x_{1}<x_{2}<\cdots<x_{N}\right\}
$$

of the standard (Cayley) graph $\mathbb{Z}^{N}$. In Section 2 we show that $H_{N}$ (when suitably restricting to $\mathbb{Z}_{<}^{N}$ ) is equal to the sum of a Toeplitz operator and a multiplicative 
potential, both belonging to the Toeplitz-like $C^{*}$-algebra $\mathscr{T}<\left(\mathbb{Z}^{N}\right)$ generated by the unilateral shifts on $\mathbb{Z}_{<}^{N}$.

Although $\mathscr{T}^{<}\left(\mathbb{Z}^{N}\right)$ is of none of the types thoroughly studied in the literature, its structure is simple enough to suggest spectral results for the operators it contains (see Section 3). Since the elements of $\mathscr{T}<\left(\mathbb{Z}^{N}\right)$ can be written as direct integrals over a torus, we are mainly concerned with spectral properties of the fibers (this presents a particular interest in the case of the one-dimensional Heisenberg model, $c f$. [RS78, Yaf00]). We express in Section 4 the essential spectrum of the fibers as a union of spectra of a family of subhamiltonians, improving part of the statements of [Zol82, which concern a larger class of operators but imposes an unnecessary exponential decay condition. We consider that our formalism and proofs are much more simple and natural than those of Zol82. In Section 15 we show the following type of result concerning the whole Hamiltonian $H_{N}$. If $\kappa$ is a continuous real function with suitable support, then there exists a natural family of multiplication operators $\left\{\chi_{n}\right\}_{n \in \mathbb{N}}$ for which $\left\|\chi_{n} \kappa\left(H_{N}\right)\right\|$ is arbitrarily small if $n$ is large enough. This can be reformulated in terms of the evolution group $\left\{\mathrm{e}^{-i t H_{N}}\right\}_{t \in \mathbb{R}}$ : at energies belonging to $\operatorname{supp}(\kappa)$, the system gouverned by $H_{N}$ stays "out of $\operatorname{supp}\left(\chi_{n}\right)$ " uniformly in time. Practically $\operatorname{supp}(\kappa)$ must not intersect the spectrum of a certain subhamiltonian associated to some ideal of $\mathscr{T}<\left(\mathbb{Z}^{N}\right)$. For the Heisenberg model we put into evidence a nice interpretation involving cluster properties of $N$-magnon states.

We think that the structure of $\mathscr{T}<\left(\mathbb{Z}^{N}\right)$ may also be crucial for proving finer spectral and scattering properties. The obtention of a Mourre estimate, which is a first step in this direction, is a problem under review.

Actually the setting generalizes from $\mathbb{Z}^{N}$ to $\left(\mathbb{Z}_{\text {lex }}^{m}\right)^{N}$, where $\mathbb{Z}_{\text {lex }}^{m}$ is the group $\mathbb{Z}^{m}$ ordered lexicographically. This relies on rather deep results of Mur91 on Toeplitz algebras associated to certain ordered groups. The statements of the present article can be pushed to this more general case; they just require more involved notations. Unfortunately, this is not the right framework to study the $m$-dimensional Heisenberg model (another type of Toeplitz algebra is needed), so we refrained from giving explicit detailed results in this situation; see however Remark 4.3..

Let us finally mention that our treatment has few direct connections with previous work on the spectral theory of Toeplitz operators. We were actually guided by the $C^{*}$-algebra approach to spectral analysis for Schrödinger operators, as in ABG96, DG00, GI02, Măn02, AMP02.

\section{The one-dimensional Heisenberg model}

In order to justify the class of operators we study, we present here briefly and rather formally the one-dimensional Heisenberg model. Further details may be found in Str67, Str74, RS78.

We consider the one-dimensional lattice $\mathbb{Z}$ with a spin- $\frac{1}{2}$ attached at each 
vertex. Let

$$
\mathbb{F}(\mathbb{Z}):=\{\alpha: \mathbb{Z} \rightarrow\{0,1\}: \operatorname{supp}(\alpha) \text { is finite }\},
$$

and write $\left\{e^{0}, e^{1}\right\}:=\{(0,1),(1,0)\}$ for the canonical basis of the spin- $\frac{1}{2}$ Hilbert space $\mathbb{C}^{2}$. For any $\alpha \in \mathbb{F}(\mathbb{Z})$ we denote by $e^{\alpha}$ the element $\left\{e^{\alpha(x)}\right\}_{x \in \mathbb{Z}}$ of the direct product $\prod_{x \in \mathbb{Z}} \mathbb{C}_{x}^{2}$. We distinguish the vector $e^{\alpha_{0}}$, where $\alpha_{0}(x):=0$ for all $x \in \mathbb{Z}$. Each element $e^{\alpha}$ is interpreted as a pure state of the system of spins, and $e^{\alpha_{0}}$ as its ground state with all spins pointing down. The Hilbert space $€$ of the system (which is spanned by the states with all but finitely many spins pointing down) is the incomplete tensor product [Str67, Sec. 2], Str74 Sec. 2]

$$
\mathrm{E}:=\bigotimes_{x \in \mathbb{Z}}^{\alpha_{0}} \mathbb{C}_{x}^{2} \equiv \text { closed span }\left\{e^{\alpha}: \alpha \in \mathbb{F}(\mathbb{Z})\right\}
$$

The dynamics of the spins is given by the nearest-neighbour Heisenberg Hamiltonian

$$
L:=-\frac{1}{2} \sum_{|x-y|=1}\left\{a\left[\sigma_{1}^{(x)} \sigma_{1}^{(y)}+\sigma_{2}^{(x)} \sigma_{2}^{(y)}\right]+b\left[\sigma_{3}^{(x)} \sigma_{3}^{(y)}-1\right]\right\} .
$$

The operator $\sigma_{j}^{(x)}$ acts in $\mathrm{E}$ as the identity operator on each factor $\mathbb{C}_{y}^{2}$, except on the component $\mathbb{C}_{x}^{2}$ where it acts as the Pauli matrix $\sigma_{j}$. The scalars $a, b \in \mathbb{R}$ prescribe the anisotropy of the system. The case $a \neq b$ corresponds to the XXZ model, whereas the standard Heisenberg model is obtained for $a=b$.

Let $\mathcal{H}:=\ell^{2}[\mathbb{F}(\mathbb{Z})]$. Then the Hilbert spaces $\mathrm{E}$ and $\mathcal{H}$ are isomorphic due to the unitarity of the mapping $\varsigma: \mathrm{L} \rightarrow \mathcal{H}$ sending $e^{\alpha}$ onto $\chi_{\{\alpha\}}$ for all $\alpha\left(\chi_{\{\alpha\}}\right.$ stands for the characteristic function of the singleton $\alpha)$. In particular, the set $\mathbb{F}(\mathbb{Z})$ may be considered as the configuration space for the system of spins. The Hamiltonian $L$ is unitarily equivalent to a difference operator in $\mathcal{H}$. Given $\alpha \in \mathbb{F}(\mathbb{Z})$ and $x \in \alpha, y \notin \alpha$, we write $\alpha_{x}^{y}$ for the function of $\mathbb{F}(\mathbb{Z})$ such that $\operatorname{supp}\left(\alpha_{x}^{y}\right)=\operatorname{supp}(\alpha) \sqcup\{y\} \backslash\{x\}$.

Lemma 2.1. For any $f \in \mathcal{H}, \alpha \in \mathbb{F}(\mathbb{Z})$, one has the equality

$$
\left(\varsigma^{-1} L \varsigma f\right)(\alpha)=-2 \sum_{|x-y|=1} \alpha(x)[1-\alpha(y)]\left[a f\left(\alpha_{x}^{y}\right)-b f(\alpha)\right] .
$$

Proof. The claim follows from a direct calculation using the properties of the Pauli matrices.

For $N \in \mathbb{N}$, let $\mathbb{F}_{N}(\mathbb{Z}):=\{\alpha \in \mathbb{F}(\mathbb{Z}): \operatorname{supp}(\alpha)$ has $N$ elements $\}$ and set $\mathcal{H}_{N}:=\ell^{2}\left[\mathbb{F}_{N}(\mathbb{Z})\right]$. Lemma 2.1 shows that the subspace $\mathcal{H}_{N}$ of $\mathcal{H}$ is left invariant by $\varsigma^{-1} L \varsigma$. This is due to the fact that the Hamiltonian $L$ commutes with the (magnon) number operator $\frac{1}{2} \sum_{x \in X}\left(\sigma_{3}^{(x)}+1\right)$. Moreover it is straightforward to show that the restriction $H_{N}:=\left(\varsigma^{-1} L \varsigma\right) \uparrow \mathcal{H}_{N}$ is bounded and symmetric.

The operator $H_{N}$ has a more convenient form in another representation, which deserves the introduction of some notations. Elements of $\mathbb{Z}^{N}$ are denoted 
generically by $\xi \equiv\left(x_{1}, \ldots, x_{N}\right), \eta \equiv\left(y_{1}, \ldots, y_{N}\right)$ or $\zeta \equiv\left(z_{1}, \ldots, z_{N}\right)$, and $P^{<}$ stands for the multiplication operator in $\ell^{2}\left(\mathbb{Z}^{N}\right)$ by the characteristic function $\chi_{\mathbb{Z}^{N}}$. We shall often interpret this projection as an operator from $\ell^{2}\left(\mathbb{Z}^{N}\right)$ to $\ell^{2}\left(\mathbb{Z}_{<}^{N}\right)$. For each $\eta \in \mathbb{Z}^{N}$ we define the unitary "bilateral shift" $u_{\eta}$ in $\ell^{2}\left(\mathbb{Z}^{N}\right)$ by

$$
\left(u_{\eta} f\right)(\xi):=f(\xi-\eta), \quad f \in \ell^{2}\left(\mathbb{Z}^{N}\right), \xi \in \mathbb{Z}^{N} .
$$

We also define the "unilateral shift" $v_{\eta}^{<}:=P^{<} u_{\eta} \uparrow \ell^{2}\left(\mathbb{Z}_{<}^{N}\right)$, which is a partial isometry in $\ell^{2}\left(\mathbb{Z}_{<}^{N}\right)$. Thus, for any $\varphi \in \ell^{1}\left(\mathbb{Z}^{N}\right)$, one can consider the convolution operator

$$
C_{\varphi}:=\sum_{\eta \in \mathbb{Z}^{N}} \varphi(\eta) u_{\eta}
$$

in $\ell^{2}\left(\mathbb{Z}^{N}\right)$, and the Toeplitz operator

$$
T_{\varphi}^{<}:=\sum_{\eta \in \mathbb{Z}^{N}} \varphi(\eta) v_{\eta}^{<}
$$

in $\ell^{2}\left(\mathbb{Z}_{<}^{N}\right)$. These operators are related by the formula $T_{\varphi}^{<}=P^{<} C_{\varphi}\left\lceil\ell^{2}\left(\mathbb{Z}_{<}^{N}\right)\right.$. Let us also consider the projections $q_{\eta}^{<}:=v_{\eta}^{<}\left(v_{\eta}^{<}\right)^{*}$ on the range of the partial isometries $v_{\eta}^{<}$. One sees easily that $q_{\eta}^{<}$is the multiplication operator by the characteristic function of the set $\mathbb{Z}_{<}^{N} \cap\left(\mathbb{Z}_{<}^{N}+\eta\right)$. Finally, among the multiplication operators in $\ell^{2}\left(\mathbb{Z}_{<}^{N}\right)$, we distinguish those of the form

$$
V_{\varphi}^{<}:=\sum_{\eta \in \mathbb{Z}^{N}} \varphi(\eta) q_{\eta}^{<}, \quad \varphi \in \ell^{1}\left(\mathbb{Z}^{N}\right) .
$$

We identify now the Heisenberg Hamiltonian to an operator of the form above. The set $S$ stands for the collection of vectors $\left\{s_{i}^{ \pm}\right\}_{i=1}^{N} \subset \mathbb{Z}^{N}$ with components $\left(s_{i}^{ \pm}\right)_{j}:= \pm \delta_{i j}$. Notice that $S$ is a symmetric family of generators for the group $\mathbb{Z}^{N}$.

Proposition 2.2. The Hamiltonian $H_{N}$ is unitarily equivalent to the operator $T_{\varphi}^{<}+V_{\psi}^{<}$, where $\varphi:=-2 a \chi_{S}$ and $\psi:=2 b \chi_{S}$.

Proof. Let $\phi: \mathbb{Z}_{<}^{N} \rightarrow \mathbb{F}_{N}(\mathbb{Z})$ be the one-to-one map $\xi \mapsto \chi_{\left\{x_{1}, \ldots, x_{N}\right\}}$. Let $\Phi: \mathcal{H}_{N} \rightarrow$ $\ell^{2}\left(\mathbb{Z}_{<}^{N}\right)$ be the unitary operator given by $\Phi(f):=f \circ \phi$ for any $f \in \mathcal{H}_{N}$. Then one has for $g \in \ell^{2}\left(\mathbb{Z}_{<}^{N}\right), \xi \in \mathbb{Z}_{<}^{N}$

$$
\left(\Phi H \Phi^{-1} g\right)(\xi)=-2 \sum_{j=1}^{N} \sum_{\substack{y=x_{j} \pm 1 \\ y \notin\left\{x_{1}, \ldots, x_{N}\right\}}}\left\{a g\left[\phi^{-1}\left(\chi_{\left\{x_{1}, \ldots, x_{j-1}, y, x_{j+1}, \ldots, x_{N}\right\}}\right)\right]-b g(\xi)\right\} .
$$

On another hand

$$
\phi^{-1}(\alpha)=(\min \alpha, \min (\alpha \backslash\{\min \alpha\}), \ldots)
$$


for any $\alpha \in \mathbb{F}_{N}(\mathbb{Z})$. Thus

$$
\Phi^{-1}\left(\chi_{\left\{x_{1}, \ldots, x_{j-1}, y, x_{j+1}, \ldots, x_{N}\right\}}\right)=\left(x_{1}, \ldots, x_{j-1}, y, x_{j+1}, \ldots, x_{N}\right)
$$

if $y=x_{j} \pm 1, y \notin\left\{x_{1}, \ldots, x_{N}\right\}$ and $x_{1}<\ldots<x_{N}$. This implies that

$$
\begin{aligned}
\left(\Phi H \Phi^{-1} g\right)(\xi) & =-2 \sum_{s \in S} \chi_{\mathbb{Z}_{<}^{N}}(\xi-s)[a g(\xi-s)-b g(\xi)] \\
& =-2 \sum_{s \in S}\left[\left(a v_{s}^{<}-b q_{s}^{<}\right) g\right](\xi) \\
& =\left[\left(T_{\varphi}^{<}+V_{\psi}^{<}\right) g\right](\xi)
\end{aligned}
$$

Proposition 2.2 is one of the motivations to study operators of the form $T_{\varphi}^{<}+V_{\psi}^{<}$for general functions $\varphi, \psi \in \ell^{1}\left(\mathbb{Z}^{N}\right)$. Actually, we can even indicate a larger class of operators $T_{\varphi}^{<}+V_{\psi}^{<}$which have an interpretation on their own, outside Toeplitz theory. If $M \subset \mathbb{Z}^{N}$ a finite subset such that $\eta \in M$ implies $-\eta \in M$, then one can associate to it a Cayley graph having $\mathbb{Z}^{N}$ as set of vertices by declaring that $\eta$ and $\zeta$ are connected iff $\eta-\zeta \in M$. The Laplace operator $\Delta^{M}$ of this Cayley graph is a convolution operator in $\ell^{2}\left(\mathbb{Z}^{N}\right)$, easy to understand when applying a Fourier transformation. On the other hand Laplacians $\Delta_{E}^{M}$ on subgraphs $E$ of this Cayley graph could be complicated objects. However it easy to show the identity $\Delta_{\mathbb{Z}_{<}^{N}}^{M}=T_{\chi_{M}}^{<}+V_{-\chi_{M}}^{<}$, which applies to the Heisenberg model in the case $M=S$. Thus the Laplacians $\Delta_{\mathbb{Z}_{<}^{N}}^{M}$ are subject of the spectral results that follow below.

\section{Toeplitz algebras}

In this section we collect some results on the Toeplitz-like algebra $\mathscr{T}<\left(\mathbb{Z}^{N}\right)$. We first introduce the appropriate abstract setting.

Let $X$ be a (discrete) abelian group and $E$ be a non-void subset of $X$. The projection $P^{E}: \ell^{2}(X) \rightarrow \ell^{2}(E)$ is defined in $\ell^{2}(X)$ as the multiplication operator by the characteristic function $\chi_{E}$. As before we introduce the unitary translation operators $\left\{u_{\eta}\right\}_{\eta \in X}$ in $\ell^{2}(X)$ and the partial isometries $\left\{v_{\eta}^{E}:=P^{E} u_{\eta}\left\lceil\ell^{2}(E)\right\}_{\eta \in X}\right.$ in $\ell^{2}(E)$. Once again, for $\varphi \in \ell^{1}(X)$, the "Toeplitz operator"

$$
T_{\varphi}^{E}:=\sum_{\eta \in X} \varphi(\eta) v_{\eta}^{E}
$$

and the "potential"

$$
V_{\varphi}^{E}:=\sum_{\eta \in X} \varphi(\eta) q_{\eta}^{E} \equiv \sum_{\eta \in X} \varphi(\eta) v_{\eta}^{E}\left(v_{\eta}^{E}\right)^{*}
$$

are available as operators in $\ell^{2}(E)$. 
Definition 3.1. The $C^{*}$-algebra $\mathscr{T}^{E}(X) \subset \mathscr{B}\left[\ell^{2}(E)\right]$ generated by the family $\left\{v_{\eta}^{E}\right\}_{\eta \in E}$ is called the Toeplitz algebra of the group $X$ with respect to the subset E.

Obviously $\mathscr{T}^{E}(X)$ contains all the operators of the form $T_{\varphi}^{E}+V_{\psi}^{E}, \varphi, \psi \in$ $\ell^{1}\left(\mathbb{Z}^{N}\right)$ (and many others). In fact $\mathscr{T}^{E}(X)$ is also generated by the family $\left\{T_{\varphi}^{E}\right.$ : $\left.\varphi \in \ell^{1}(X)\right\}$ as in the case of usual Toeplitz algebras. If $E=X, \mathscr{T}^{E}(X)$ is equal to $C_{\mathrm{r}}^{*}(X)$, the reduced group $C^{*}$-algebra of $X$. Since $X$ is abelian, we may identify it with $C^{*}(X)$, the envelopping $C^{*}$-algebra of the convolution Banach $*$-algebra $\ell^{1}(X)$. The identification puts into correspondence $u_{\eta}$ with $\delta_{\eta}:=\chi_{\{\eta\}}$. Due to the Fourier transform, $C^{*}(X)$ is isomorphic to the $C^{*}$-algebra $C(\widehat{X})$ of continuous complex functions on $\widehat{X}$, where $\widehat{X}$ is the (compact, abelian) dual group of $X$. The conventions "in dimension zero" are clear: The group is $X=\{0\}$, with subset $E=\{0\}$, all functions are scalars, and for $\varphi \in \mathbb{C}$ one sets $T_{\varphi}=V_{\varphi}:=\varphi 1$ in $\ell^{2}(X) \equiv \mathbb{C}$.

The algebras $\mathscr{T}^{E}(X)$ were mainly studied for $X$ an ordered group and $E$ its positive cone Mur87, Mur91, Xu99, [XC99. The standard case is the "classical" Toeplitz algebra $\mathscr{T}^{\mathbb{N}}(\mathbb{Z}) \equiv \mathscr{T}$ Dou72] associated to the unilateral shift on $\ell^{2}(\mathbb{N})$ (often presented in a Fourier transformed realization). In our proofs the isomorphic algebra $\mathscr{T}^{*}:=\mathscr{T}^{\mathbb{N}^{*}}(\mathbb{Z}), \mathbb{N}^{*}:=\{1,2, \ldots\}$, will appear more naturally. The case $X=\mathbb{Z}^{2}, E=\mathbb{N}^{2}$ is studied in [DH71]. The most relevant Toeplitz algebra for us is $\mathscr{T}^{<}\left(\mathbb{Z}^{N}\right):=\mathscr{T}^{\mathbb{Z}^{N}}\left(\mathbb{Z}^{N}\right)$, which is not of ordered type. We shall point out its structure, which will be the main tool in analysing the operators $T_{\varphi}^{<}+V_{\psi}^{<}$.

The key facts are:

(A) If $\theta: X \rightarrow X^{\prime}$ is a group isomorphism sending $E$ onto $E^{\prime}$, then $\mathscr{T}^{E}(X)$ and $\mathscr{T}^{E^{\prime}}\left(X^{\prime}\right)$ are naturally isomorphic, the element $v_{\eta}^{E}$ of $\mathscr{T}^{E}(X)$ being sent onto $v_{\theta(\eta)}^{E^{\prime}} \in \mathscr{T}^{E^{\prime}}\left(X^{\prime}\right)$.

(B) If $E_{j}$ is a subset of a group $X_{j}, j=1, \ldots, m$, then $\mathscr{T}^{E_{1} \times \cdots \times E_{m}}(X)$ can be identified with the spatial tensor product $C^{*}$-algebra $\bigotimes_{j=1}^{m} \mathscr{T}^{E_{j}}\left(X_{j}\right)$, $v_{\left(\eta_{1}, \ldots, \eta_{m}\right)}^{E}$ being identified with $\otimes_{j=1}^{m} v_{\eta_{j}}^{E_{j}}$.

Both isomorphisms are unitarily implemented, but this will not be used explicitly in the sequel.

Let $\theta: \mathbb{Z}^{N} \rightarrow \mathbb{Z}^{N}$ be the group automorphism defined by

$$
\theta\left(y_{1}, \ldots, y_{N}\right):=\left(y_{1}, y_{2}-y_{1}, \ldots, y_{N}-y_{N-1}\right),
$$

with inverse

$$
\theta^{-1}\left(z_{1}, \ldots, z_{N}\right)=\left(z_{1}, z_{1}+z_{2}, \ldots, z_{1}+\cdots+z_{N}\right) .
$$

Obviously, $\mathcal{E}:=\theta\left(\mathbb{Z}_{<}^{N}\right)=\mathbb{Z} \times\left(\mathbb{N}^{*}\right)^{N-1}$. Thus, by $(\mathrm{A}), \mathscr{T}^{<}\left(\mathbb{Z}^{N}\right)$ and $\mathscr{T}^{\mathcal{E}}\left(\mathbb{Z}^{N}\right)$ are isomorphic, $v_{\eta}^{<}$being sent onto $v_{\theta(\eta)}^{\mathcal{E}}$ for any $\eta \in \mathbb{Z}^{N}$. Consequently this isomorphism sends $T_{\varphi}^{<}$onto $T_{\varphi \circ \theta^{-1}}^{\mathcal{E}}$ and $V_{\psi}^{<}$onto $V_{\psi \circ \theta^{-1}}^{\mathcal{E}}$. In the next few lines we only consider the case of $T_{\varphi}^{<}$. 
By applying $(\mathrm{B})$, one gets an isomorphism between $\mathscr{T}^{\mathcal{E}}\left(\mathbb{Z}^{N}\right)$ and $\mathscr{T}^{\mathbb{Z}}(\mathbb{Z}) \otimes$ $\left[\mathscr{T}^{\mathbb{N}^{*}}(\mathbb{Z})\right]^{\otimes(N-1)} \equiv C^{*}(\mathbb{Z}) \otimes\left(\mathscr{T}^{*}\right)^{\otimes(N-1)}$, under which $T_{\varphi \circ \theta^{-1}}^{\mathcal{E}}$ is transformed into

$$
\sum_{\left(z_{1}, \ldots, z_{N}\right) \in \mathbb{Z}^{N}}\left(\varphi \circ \theta^{-1}\right)\left(z_{1}, \ldots, z_{N}\right) \delta_{z_{1}} \otimes v_{z_{2}}^{\mathbb{N}^{*}} \otimes \cdots \otimes v_{z_{N}}^{\mathbb{N}^{*}} .
$$

Now we apply the partial Fourier transform on the first variable. It maps $\delta_{z_{1}} \in$ $C^{*}(\mathbb{Z})$ onto $\mathrm{e}_{z_{1}} \in C(\mathbb{T})$, where $\mathbb{T}$ is equal to the interval $[0,1]$ (with 0 identified to 1) and $\mathrm{e}_{z}(\tau):=\mathrm{e}^{-2 \pi i z \tau}$ for all $z \in \mathbb{Z}, \tau \in \mathbb{T}$. Namely we use $\mathscr{F}_{1}:=\mathscr{F} \otimes 1$, where $(\mathscr{F} f)(\tau):=\sum_{z \in \mathbb{Z}} \mathrm{e}_{z}(\tau) f(z)$ for all $\tau \in \mathbb{T}, f \in \ell^{1}(\mathbb{Z})$. When performing the sum over $z_{1}$, the operator (3.1) becomes

$$
\sum_{\left(z_{2}, \ldots, z_{N}\right) \in \mathbb{Z}^{N-1}}\left[\mathscr{F}_{1}\left(\varphi \circ \theta^{-1}\right)\right]\left(\cdot, z_{2}, \ldots, z_{N}\right) v_{z_{2}}^{\mathbb{N}^{*}} \otimes \cdots \otimes v_{z_{N}}^{\mathbb{N}^{*}},
$$

which will be regarded as an element of $C(\mathbb{T}) \otimes \mathscr{T}^{\otimes(N-1)} \equiv C\left[\mathbb{T} ; \mathscr{T}^{\otimes(N-1)}\right]$. Similar arguments can be carried on for $V_{\psi}^{<}$. When summing up we get the following lemma. For any $\rho \in \ell^{1}\left(\mathbb{Z}^{N}\right)$ and $\tau \in \mathbb{T}$ we define $\mu(\tau) \rho \in \ell^{1}\left(\mathbb{Z}^{N-1}\right)$ by

$$
[\mu(\tau) \rho]\left(z_{2}, \ldots, z_{N}\right):=\left[\mathscr{F}_{1}\left(\rho \circ \theta^{-1}\right)\right]\left(\tau, z_{2}, \ldots, z_{N}\right) .
$$

Lemma 3.2. The $C^{*}$-algebras $\mathscr{T}<\left(\mathbb{Z}^{N}\right)$ and $C(\mathbb{T}) \otimes \mathscr{T}^{\otimes(N-1)}$ are naturally isomorphic. The isomorphism sends $T_{\varphi}+V_{\psi}$ onto the direct integral

$$
\int_{\mathbb{T}}^{\oplus} \mathrm{d} \tau\left(T_{\mu(\tau) \varphi}^{N-1}+V_{\mu(0) \psi}^{N-1}\right)
$$

where the exponent of the Toeplitz operators refers to the subset $\left(\mathbb{N}^{*}\right)^{N-1}$ of the group $\mathbb{Z}^{N-1}$.

The presence of a direct integral is connected to the invariance of our operators under a natural action of $\mathbb{Z}$ on $\mathbb{Z}_{<}^{N}$ by translations. For the Heisenberg model, this can be traced back to any of the earlier representations of the Hamiltonian.

As a rule, the spectral results will be stated only for operators of the form $T_{\varphi}^{<}+V_{\psi}^{<}$. By introducing suitable notations, they could be extended to all the elements of $\mathscr{T}<\left(\mathbb{Z}^{N}\right)$.

Corollary 3.3. For any real functions $\varphi, \psi \in \ell^{1}\left(\mathbb{Z}^{N}\right)$, one has

$$
\sigma_{\mathrm{ess}}\left(T_{\varphi}^{<}+V_{\psi}^{<}\right)=\sigma\left(T_{\varphi}^{<}+V_{\psi}^{<}\right)=\bigcup_{\tau \in \mathbb{T}} \sigma\left(T_{\mu(\tau) \varphi}^{N-1}+V_{\mu(0) \psi}^{N-1}\right)
$$

Proof. The essential spectrum of an element $A$ of a $C^{*}$-algebra $\mathscr{C}$ composed of bounded operators in a Hilbert space $\mathcal{G}$ coincides with the spectrum of its image in the quotient $\mathscr{C} /[\mathscr{C} \cap \mathscr{K}(\mathcal{G})]$, where $\mathscr{K}(\mathcal{G})$ denotes the compact operators in $\mathcal{G}$. Thus the first equality follows from the obvious fact that there is no non-trivial compact 
operator in $C(\mathbb{T}) \otimes \mathscr{T} \otimes(N-1) \subset \mathscr{B}\left[\mathrm{L}^{2}(\mathbb{T})\right] \otimes \mathscr{B}\left[\ell^{2}\left(\mathbb{N}^{*}\right)\right]^{\otimes(N-1)} \subset \mathscr{B}\left[\ell^{2}\left(\mathbb{T} \times\left(\mathbb{N}^{*}\right)^{N-1}\right)\right]$. For the second equality, we apply Lemma 3.2 and the discussion in ABG96. Sec. 8.2.4] on the spectrum of observables defined by a continuous family of selfadjoint operators. Note that the union on the r.h.s. is automatically closed.

If $N=1$, then $T_{\varphi}^{<}$is the convolution operator $C_{\varphi}$ and $V_{\psi}^{<}$is the multiplication operator by $\sum_{z \in \mathbb{Z}} \psi(z)=(\mathscr{F} \psi)(0) \in \mathbb{R}$. Thus the spectrum (3.3) reduces to

$$
\sigma\left(T_{\varphi}^{<}+V_{\psi}^{<}\right)=(\mathscr{F} \varphi)(\mathbb{T})+(\mathscr{F} \psi)(0)=[\min (\mathscr{F} \varphi), \max (\mathscr{F} \varphi)]+(\mathscr{F} \psi)(0)
$$

as it should be.

\section{The essential spectrum of the fiber Hamiltoni- ans}

The fiber Hamiltonians $H(\tau):=T_{\mu(\tau) \varphi}^{N-1}+V_{\mu(0) \psi}^{N-1}$ can be interpreted physically as "energy operators at fixed quasi-momentum $\tau$ ". We study now their esssential spectrum. We use again the well known fact that, given a Hilbert space $\mathcal{G}$, the essential spectrum of an operator $A \in \mathscr{B}(\mathcal{G})$ coincides with the spectrum of its image in the Calkin algebra $\mathscr{B}(\mathcal{G}) / \mathscr{K}(\mathcal{G})$, where $\mathscr{K}(\mathcal{G})$ is the ideal of compact operators.

The fibers $H(\tau)$ act in $\ell^{2}\left[\left(\mathbb{N}^{*}\right)^{N-1}\right] \simeq \ell^{2}\left(\mathbb{N}^{*}\right)^{\otimes(N-1)}$ and belong to the $C^{*}$ algebra $\left(\mathscr{T}^{*}\right)^{\otimes(N-1)}$. So we are faced to the problem of understanding the quotient of $\left(\mathscr{T}^{*}\right)^{\otimes(N-1)}$ by $\mathscr{K}\left[\ell^{2}\left(\mathbb{N}^{*}\right)^{\otimes(N-1)}\right]$, the latter being identified to $\left(\mathscr{K}^{*}\right)^{\otimes(N-1)}$, where $\mathscr{K}^{*}:=\mathscr{K}\left[\ell^{2}\left(\mathbb{N}^{*}\right)\right]$. The discussion in [DH71] is relevant here, especially in the case $N=2$. We start by proving a result in a more abstract setting.

Lemma 4.1. Let $\mathscr{C}_{j}$ be a nuclear $C^{*}$-subalgebra of $\mathscr{B}\left(\mathcal{H}_{j}\right)$, where $\mathcal{H}_{j}$ is a Hilbert space, $j=1,2$. Let $\mathscr{I}_{j}$ be an ideal of $\mathscr{C}_{j}$ and let $\pi_{j}: \mathscr{C}_{j} \rightarrow \mathscr{C}_{j} / \mathscr{I}_{j}$ be the canonical *-morphism. Then the mapping

$$
\begin{aligned}
\bar{\pi}: \mathscr{C}_{1} \otimes \mathscr{C}_{2} & \rightarrow\left[\left(\mathscr{C}_{1} / \mathscr{I}_{1}\right) \otimes \mathscr{C}_{2}\right] \oplus\left[\mathscr{C}_{1} \otimes\left(\mathscr{C}_{2} / \mathscr{I}_{2}\right)\right] \\
A & \mapsto\left\{\left(\pi_{1} \otimes 1\right)(A),\left(1 \otimes \pi_{2}\right)(A)\right\}
\end{aligned}
$$

is a $*$-morphism, and $\operatorname{ker}(\bar{\pi})=\mathscr{I}_{1} \otimes \mathscr{I}_{2}$.

Proof. Since $\mathscr{C}_{1}$ and $\mathscr{C}_{2}$ are nuclear, the mappings $\pi_{1} \otimes 1$ and $1 \otimes \pi_{2}$ are (surjective) $*$-morphisms with $\operatorname{ker}\left(\pi_{1} \otimes 1\right)=\mathscr{I}_{1} \otimes \mathscr{C}_{2}$ and $\operatorname{ker}\left(1 \otimes \pi_{2}\right)=\mathscr{C}_{1} \otimes \mathscr{I}_{2}$ Mur90. Thm. 6.5.2]. From this it follows that $\bar{\pi}$ is a $*$-morphism with $\operatorname{ker}(\bar{\pi})=\left(\mathscr{I}_{1} \otimes \mathscr{C}_{2}\right) \cap$ $\left(\mathscr{C}_{1} \otimes \mathscr{I}_{2}\right)$. Since ideals in nuclear $C^{*}$-algebras are nuclear, the triple $\left(\mathscr{I}_{1}, \mathscr{C}_{2}, \mathscr{I}_{2}\right)$ verifies the (right) slice map conjecture Was78 Prop. 10]. Thus one gets the equality $\operatorname{ker}(\bar{\pi})=\mathscr{I}_{1} \otimes \mathscr{I}_{2}$ by an easy adaptation of the proof of Was76. Cor. $5]$. 
We need two more notations. For any $j \in\{2, \ldots, N\}, \varrho \in \ell^{1}\left(\mathbb{Z}^{N-1}\right)$ and $\tau \in \mathbb{T}$, we define $\nu_{j}(\tau) \varrho \in \ell^{1}\left(\mathbb{Z}^{N-2}\right)$ by

$$
\left[\nu_{j}(\tau) \varrho\right]\left(z_{2}, \ldots, z_{j-1}, z_{j+1}, \ldots, z_{N}\right):=\left(\mathscr{F}_{j} \varrho\right)\left(z_{2}, \ldots, z_{j-1}, \tau, z_{j+1}, \ldots, z_{N}\right),
$$

$\mathscr{F}_{j}$ being the Fourier transformation in the $j^{\text {th }}$ variable. Furthermore we denote by $\Sigma_{j}\left(\tau, \tau^{\prime}\right)$ the spectrum of the Toeplitz operator (relative to the pair $\left.\left\{\mathbb{Z}^{N-2},\left(\mathbb{N}^{*}\right)^{N-2}\right\}\right)$

$$
T_{\nu_{j}\left(\tau^{\prime}\right) \mu(\tau) \varphi}^{N-2}+V_{\nu_{j}(0) \mu(0) \psi}^{N-2}
$$

acting in $\ell^{2}\left[\left(\mathbb{N}^{*}\right)^{N-2}\right]$.

Theorem 4.2. Let $\varphi, \psi \in \ell^{1}\left(\mathbb{Z}^{N}\right)$ be real functions and $\tau \in \mathbb{T}$. Then one has

$$
\sigma_{\text {ess }}\left(T_{\mu(\tau) \varphi}^{N-1}+V_{\mu(0) \psi}^{N-1}\right)=\bigcup_{j=2}^{N} \bigcup_{\tau^{\prime} \in \mathbb{T}} \Sigma_{j}\left(\tau, \tau^{\prime}\right) .
$$

Proof. By analogy to (the Fourier transformed version of) the isomorphism $\mathscr{T} / \mathscr{K}\left[\ell^{2}(\mathbb{N})\right] \simeq C(\mathbb{T})$ Dou72, Thm. 7.23], one has a canonical isomorphism $\mathscr{T}^{*} / \mathscr{K}^{*} \simeq C(\mathbb{T})$, uniquely defined by the fact that for any $z \in \mathbb{Z}$ the operator $v_{z}^{\mathbb{N}^{*}} \in \mathscr{T}^{*}$ is sent onto the function $\mathrm{e}_{z} \in C(\mathbb{T})$. Therefore the class of operators $T_{f}^{\mathbb{N}^{*}} \in \mathscr{T}^{*}, f \in \ell^{1}(\mathbb{Z})$, must be sent onto the class of functions $\mathscr{F} f \in C(\mathbb{T})$.

The Toeplitz algebra $\mathscr{T}^{*}$ is nuclear, since it is the extension of the abelian quotient $\mathscr{T}^{*} / \mathscr{K}^{*} \simeq C(\mathbb{T})$ by the nuclear ideal $\mathscr{K}^{*}$. So we may use the analog of Lemma 4.1 for the $N-1$ factors $\mathscr{C}_{2}=\ldots=\mathscr{C}_{N}=\mathscr{T}^{*}$ and $\mathscr{I}_{2}=\ldots=\mathscr{I}_{N}=\mathscr{K}^{*}$. We get an injective $*$-morphism

$$
\begin{aligned}
\left(\mathscr{T}^{*}\right)^{\otimes(N-1)} /\left(\mathscr{K}^{*}\right)^{\otimes(N-1)} \hookrightarrow & \oplus_{j=2}^{N}\left(\mathscr{T}_{*}^{\otimes(j-2)} \otimes C(\mathbb{T}) \otimes \mathscr{T}_{*}^{\otimes(N-j)}\right) \\
& \simeq \oplus_{j=2}^{N}\left(C(\mathbb{T}) \otimes \mathscr{T}_{*}^{\otimes(N-2)}\right),
\end{aligned}
$$

sending $T_{\mu(\tau) \varphi}+V_{\mu(0) \psi}$ onto the collection

$$
\left\{\int_{\mathbb{T}}^{\oplus} \mathrm{d} \tau^{\prime}\left(T_{\nu_{j}\left(\tau^{\prime}\right) \mu(\tau) \varphi}^{N-2}+V_{\nu_{j}(0) \mu(0) \psi}^{N-2}\right)\right\}_{j=2, \ldots, N} .
$$

One concludes by using ABG96 Sec. 8.2.4].

As an example, if $N=2$, one has

$$
\sigma_{\text {ess }}\left(T_{\mu(\tau) \varphi}^{1}+V_{\mu(0) \psi}^{1}\right)=\left\{\left(\mathscr{F}_{1} \mathscr{F}_{2} \varphi\right)\left(\tau-\tau^{\prime}, \tau^{\prime}\right)+\left(\mathscr{F}_{1} \mathscr{F}_{2} \psi\right)(0,0): \tau^{\prime} \in \mathbb{T}\right\},
$$

In the case of the Heisenberg model (see Proposition [2.2), one has to take $\varphi=$ $-2 a \chi_{S}$ and $\psi=2 b \chi_{S}$. Since $\left(\mathscr{F}_{1} \mathscr{F}_{2} \chi_{S}\right)\left(\tau-\tau^{\prime}, \tau^{\prime}\right)=2 \cos \left(2 \pi \tau^{\prime}\right)+2 \cos [2 \pi(\tau-$ $\left.\tau^{\prime}\right)$ ], the essential spectrum of the fiber operator coincides with the interval $\{8 b-$ $\left.4 a \cos \left(2 \pi \tau^{\prime}\right)-4 a \cos \left[2 \pi\left(\tau-\tau^{\prime}\right)\right]: \tau^{\prime} \in[0,1]\right\}$. 
Remark 4.3. Results of this section (and also of the next one) can easily be generalized to Toeplitz-like operators $T_{\varphi}^{<}+V_{\psi}^{<}$acting on the subset

$$
E=\left(\mathbb{Z}_{\operatorname{lex}}^{m}\right)_{<}^{N}:=\left\{\left(x_{1}, \ldots, x_{N}\right) \in\left(\mathbb{Z}_{\operatorname{lex}}^{m}\right)_{<}^{N}: x_{1}<x_{2}<\cdots<x_{N}\right\}
$$

of $X=\left(\mathbb{Z}_{\text {lex }}^{m}\right)^{N}$, where $\mathbb{Z}_{\text {lex }}^{m}$ is the group $\mathbb{Z}^{m}$ with the lexicographic order. In analysing the structure of $\mathcal{T}^{E}(X)$ for this case, the Toeplitz algebra $\mathscr{T}$ should be

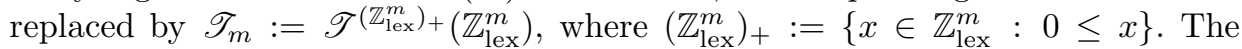
only important change concerns the calculation of the quotients $\mathscr{T}_{m} / \mathscr{K}_{m}$, where $\mathscr{K}_{m}:=\mathscr{K}\left\{\ell^{2}\left[\left(\mathbb{Z}_{\text {lex }}^{m}\right)_{+}\right]\right\}$. Namely one has to call for the rather deep result Mur91 Thm. 2.3], which implies that $\mathscr{T}_{m} / \mathscr{K}_{m}$ and $\mathscr{T}_{m-1} \otimes C(\mathbb{T})$ are $*$-isomorphic.

\section{$5 \quad$ Localization}

In the sequel we determine localization properties of the operators $T_{\varphi}^{<}+V_{\psi}^{<}$by adapting to the Toeplitz algebra $\mathscr{T}^{<}\left(\mathbb{Z}^{N}\right)$ a technique developed in [AMP02] (see also Măn03) for crossed product $C^{*}$-algebras, with applications to Schrödinger operators in $\mathbb{R}^{n}$.

Let $H$ be a selfadjoint operator in $\ell^{2}\left(\mathbb{Z}_{<}^{N}\right)$ (or in some other $\mathrm{L}^{2}$-space) and $\chi$ a non-trivial multiplication operator (for example the characteristic function of a set having a strictly positive measure). If $\kappa$ is a continuous function with support intersecting the spectrum of $H$, the operator $\chi \kappa(H)$ has no reason to be small in general. The unique a priori bound would be

$$
\|\chi \kappa(H)\| \leq\|\chi\|_{\infty} \sup _{\lambda \in \sigma(H)}|\kappa(\lambda)| .
$$

We are going to correlate $\chi$ to $\kappa$ in such a way to make the norm small without asking any of the two factors on the r.h.s. of (5.1) to be small.

Theorem 5.1. Fix $j \in\{2, \ldots, N\}$ and let $\varphi, \psi$ be real elements of $\ell^{1}\left(\mathbb{Z}^{N}\right)$. For any $n \in \mathbb{N}$ set

$$
\Omega_{j}(n):=\left\{\left(y_{1}, \ldots, y_{N}\right) \in \mathbb{Z}_{<}^{N}: y_{j}-y_{j-1} \geq n\right\} .
$$

Let $\kappa: \mathbb{R} \rightarrow \mathbb{R}$ be a continuous function with

$$
\operatorname{supp} \kappa \cap\left[\bigcup_{\tau, \tau^{\prime} \in \mathbb{T}^{2}} \Sigma_{j}\left(\tau, \tau^{\prime}\right)\right]=\varnothing .
$$

Then for each $\varepsilon>0$ there exists $n_{\varepsilon} \in \mathbb{N}$ such that

$$
\left\|\chi_{\Omega_{j}(n)} \kappa\left(T_{\varphi}^{<}+V_{\psi}^{<}\right)\right\| \leq \varepsilon
$$

for each $n \geq n_{\varepsilon}$. 
Proof. Denote by $T_{\varphi \circ \theta^{-1}}^{\mathcal{E}}+V_{\psi \circ \theta^{-1}}^{\mathcal{E}}$ the image of $T_{\varphi}^{<}+V_{\psi}^{<}$through the isomorphism $\mathscr{T}^{<}\left(\mathbb{Z}^{N}\right) \simeq C(\mathbb{T}) \otimes\left(\mathscr{T}^{*}\right)^{\otimes(N-1)}$, defined by the change of variables $\theta$. One verifies easily that the estimate (5.2) is equivalent to

$$
\left\|\chi_{\widetilde{\Omega}_{j}(n)} \kappa\left(T_{\varphi \circ \theta^{-1}}^{\mathcal{E}}+V_{\psi \circ \theta^{-1}}^{\mathcal{E}}\right)\right\| \leq \varepsilon
$$

where $\widetilde{\Omega_{j}}(n):=\theta\left[\Omega_{j}(n)\right]=\left\{\left(z_{1}, \ldots, z_{N}\right) \in \mathbb{Z} \times\left(\mathbb{N}^{*}\right)^{(N-1)}: z_{j} \geq n\right\}$. Moreover the operator $\kappa\left(T_{\varphi \circ \theta^{-1}}^{\mathcal{E}}+V_{\psi \circ \theta^{-1}}^{\mathcal{E}}\right)$ belongs to the ideal

$$
\mathscr{I}_{j}:=C(\mathbb{T}) \otimes\left(\mathscr{T}^{*}\right)^{\otimes(j-2)} \otimes \mathscr{K}^{*} \otimes\left(\mathscr{T}^{*}\right)^{\otimes(N-j)}
$$

of $\mathscr{C}:=C(\mathbb{T}) \otimes\left(\mathscr{T}^{*}\right)^{\otimes(N-1)}$. Indeed the image of $T_{\varphi \circ \theta^{-1}}^{\mathcal{E}}+V_{\psi \circ \theta^{-1}}^{\mathcal{E}}$ in the quotient

$$
\mathscr{C} / \mathscr{I}_{j} \simeq C(\mathbb{T}) \otimes\left(\mathscr{T}^{*}\right)^{\otimes(j-2)} \otimes C(\mathbb{T}) \otimes\left(\mathscr{T}^{*}\right)^{\otimes(N-j)} \simeq C(\mathbb{T})^{\otimes 2} \otimes\left(\mathscr{T}^{*}\right)^{\otimes(N-2)}
$$

is

$$
\int_{\mathbb{T}^{2}}^{\oplus} \mathrm{d} \tau \mathrm{d} \tau^{\prime}\left(T_{\nu_{j}\left(\tau^{\prime}\right) \mu(\tau) \varphi}^{N-2}+V_{\nu_{j}(0) \mu(0) \psi}^{N-2}\right)
$$

with spectrum $\bigcup_{\tau, \tau^{\prime} \in \mathbb{T}} \Sigma_{j}\left(\tau, \tau^{\prime}\right) \equiv \sigma_{\mathscr{I}_{j}}\left(T_{\varphi \circ \theta^{-1}}^{\mathcal{E}}+V_{\psi \circ \theta^{-1}}^{\mathcal{E}}\right)$. Thus, since $\operatorname{supp}(\kappa) \cap$ $\left[\bigcup_{\tau, \tau^{\prime} \in \mathbb{T}} \Sigma_{j}\left(\tau, \tau^{\prime}\right)\right]=\varnothing$, it follows by [AMP02, Lemma 1] that $\kappa\left(T_{\varphi \circ \theta^{-1}}^{\mathcal{E}}+V_{\psi \circ \theta^{-1}}^{\mathcal{E}}\right) \in$ $\mathscr{I}_{j}$. Now

$$
\chi_{\widetilde{\Omega}_{j}(n)}=1_{j-1} \otimes \chi_{\left\{z_{j} \geq n\right\}} \otimes 1_{N-j},
$$

where $\chi_{\left\{z_{j} \geq n\right\}}$ converges strongly to 0 in $\mathscr{B}\left[\ell^{2}\left(\mathbb{N}^{*}\right)\right]$ as $n \rightarrow \infty$. Thus, by examining the structure of $\mathcal{J}_{j}$, one gets

$$
\left\|\chi_{\widetilde{\Omega_{j}}(n)} \kappa\left(T_{\varphi \circ \theta^{-1}}^{\mathcal{E}}+V_{\psi \circ \theta^{-1}}^{\mathcal{E}}\right)\right\| \longrightarrow 0
$$

as $n \rightarrow \infty$.

Let $H$ be a selfadjoint operator in a Hilbert space $\mathcal{G}$ with spectral measure $E^{H}$, and let $f \in \mathcal{G}$ be an arbitrary vector. We call spectral support of $f$ with respect to $H$, and write $\operatorname{supp}(f ; H)$, for the smallest closed subset $F$ of $\mathbb{R}$ such that $E^{H}(F) f=f$. Alternatively one can characterize $\operatorname{supp}(f ; H)$ as follows:

$$
\lambda \notin \operatorname{supp}(f ; H) \text { iff } \exists \epsilon>0 \text { such that } E^{H}(\lambda-\epsilon, \lambda+\epsilon) f=0 .
$$

Obviously one has $\operatorname{supp}(f ; H) \subset \sigma(H)$. If $H$ is the Hamilton operator describing some quantum system in $\mathcal{G}$, we say that $f$ is a state with energy in $\operatorname{supp}(f ; H)$.

Corollary 5.2. Let $j, \varphi, \psi$ and $\kappa$ be as in Theorem 5.1. Then, for any $\varepsilon>0$, there exists $n_{\varepsilon} \in \mathbb{N}$ such that

$$
\left\|\chi_{\Omega_{j}(n)} \mathrm{e}^{-i t\left(T_{\varphi}^{<}+V_{\psi}^{<}\right)} f\right\| \leq \varepsilon\|f\|
$$

for all $n \geq n_{\varepsilon}, t \in \mathbb{R}$ and all $f \in \ell^{2}\left(\mathbb{Z}_{<}^{N}\right)$ satisfying

$$
\operatorname{supp}\left(f ; T_{\varphi}^{<}+V_{\psi}^{<}\right) \cap\left[\bigcup_{\tau, \tau^{\prime} \in \mathbb{T}} \Sigma_{j}\left(\tau, \tau^{\prime}\right)\right]=\varnothing .
$$


Corollary 5.2 follows trivially from Theorem 5.1 We put it into evidence for its physical interpretation in the case of the one-dimensional Heisenberg model: Intuitively, if $f$ is a normalized initial state with energy outside $\cup_{\tau, \tau^{\prime}} \Sigma_{j}\left(\tau, \tau^{\prime}\right)$, the decomposition of the system into two clusters of spins pointing up, one "at the left", composed of $j-1$ elements, and the other one "at the right", composed of $N-j+1$ elements, is highly unprobable uniformly in time if the distance $n$ between the clusters is large enough.

It is obvious that several variants are available. One can consider ideals smaller than $\mathscr{I}_{j}$, by collapsing more than one factor to the ideal of compact operators in $\ell^{2}\left(\mathbb{N}^{*}\right)$. In this way, one gets more detailed clustering information for the one-dimensional Heisenberg model, but for a priori smaller sets of energy values. The fiber Hamiltonians $T_{\mu(\tau) \varphi}^{N-1}+V_{\mu(0) \psi}^{N-1}$ can be studied identically by using ideals in the $C^{*}$-algebra $\left(\mathscr{T}^{*}\right)^{\otimes(N-1)}$.

From Corollary 3.3 and Theorem 4.2 we know that

$$
\bigcup_{j=2}^{N} \bigcup_{\tau, \tau^{\prime} \in \mathbb{T}} \Sigma_{j}\left(\tau, \tau^{\prime}\right)=\bigcup_{\tau \in \mathbb{T}} \sigma_{\mathrm{ess}}\left(T_{\mu(\tau) \varphi}^{N-1}+V_{\mu(0) \psi}^{N-1}\right) \subset \sigma\left(T_{\varphi}^{<}+V_{\psi}^{<}\right) .
$$

It does not seem easy to determine under which conditions there is room in the spectrum of $T_{\varphi}^{<}+V_{\psi}^{<}$outside $\bigcup_{\tau, \tau^{\prime} \in \mathbb{T}} \Sigma_{j}\left(\tau, \tau^{\prime}\right)$ for a given $j$. However one may expect it is often the case.

\section{Acknowledgements}

We are grateful to R. Purice for his helpful remarks. M. Măntoiu acknowledges partial support from the contract CERES, 4-187/2004. R. Tiedra de Aldecoa is supported by the Swiss National Science Foundation. Part of this work has been completed while M. Măntoiu visited the University of Geneva; he expresses his gratitude to W. Amrein for his kind hospitality.

\section{References}

[ABG96] W. O. Amrein, A. Boutet de Monvel, and V. Georgescu, $C_{0}$-groups, commutator methods and spectral theory of $N$-body hamiltonians, Progress in Math., vol. 135, Birkhäuser, Basel, 1996.

[DG00] M. Damak, V. Georgescu, $C^{*}$-cross products and a generalized mechanical N-body problem, Electron. J. Differ. Equ. Conf. 4 (2000), 51-69.

[AMP02] W. O. Amrein, M. Măntoiu, and R. Purice, Propagation properties for Schrödinger operators affiliated with certain $C^{*}$-algebras, Ann. Henri Poincaré 3 (2002), no. 6, 1215-1232. 
[Dou72] R. G. Douglas, Banach algebra techniques in operator theory, Academic Press, New York, 1972.

[DH71] R. G. Douglas and R. Howe, On the $C^{*}$-algebra of Toeplitz operators on the quarter plane, Trans. of the AMS 158 (1971), no. 1, 203-217.

[GI02] V. Georgescu and A. Iftimovici, Crossed products of $C^{*}$-algebras and spectral analysis of quantum Hamiltonians, Comm. Math. Phys. 228 (2002), no. 3, 519-560.

[Măn02] M. Măntoiu, $C^{*}$-algebras, dynamical systems at infinity and the essential spectrum of generalized Schrödinger operators, J. Reine Angew. Math. $\mathbf{5 5 0}$ (2002), 211-229.

[Măn03] _,$C^{*}$-algebras, dynamical systems, spectral analysis, Operator algebras and mathematical physics (Constanţa, 2001) (Bucharest), Theta, 2003, pp. 299-314.

[Mur87] G. J. Murphy, Ordered groups and Toeplitz algebras, J. Operator Theory 18 (1987), 303-326.

[Mur90] $\longrightarrow C^{*}$-algebras and operator theory, Academic Press, Inc., Boston, 1990.

[Mur91] - Toeplitz operators and algebras, Math. Z. 208 (1991), 355-362.

[RS78] M. Reed and B. Simon, Methods of modern mathematical physics, I-IV, Academic Press, New York, 1972-1978.

[Str67] R. F. Streater, The Heisenberg ferromagnet as a quantum field theory, Comm. Math. Phys. 6 (1967), 233-247.

[Str74] _ Spin-wave scattering, Scattering theory in mathematical physics (Dordrecht) (J. A. Lavita and J. P. Marchand, eds.), D. Reidel, 1974.

[Was76] S. Wassermann, The slice map problem for $C^{*}$-algebras, Proc. London Math. Soc. 32 (1976), no. 3, 537-559.

[Was78] A pathology in the ideal space of $L(H) \otimes L(H)$, Indiana Univ. Math. J. 27 (1978), no. 6, 1011-1020.

[Xu99] Q. Xu, Toeplitz algebras and discrete abelian quasily odered groups, Proc. of the AMS 128 (1999), no. 5, 1405-1408.

[XC99] Q. Xu and X. Chen, Toeplitz $C^{*}$-algebras on ordered groups and their ideals of finite elements, Proc. of the AMS 127 (1999), no. 2, 553-561.

[Yaf00] D. Yafaev, Scattering theory: some old and new problems, Lecture Notes in Mathematics, vol. 1735, ch. 16, Springer-Verlag, Berlin, 2000. 
[Zol82] H. Zoladek, The essential spectrum of an $N$-particle additive cluster operator, English translation in Theoret. and Math. Phys 53 (1982), no. $2,216-226$. 